\title{
Effective interactions of knotted ring polymers
}

Arturo Narros, ${ }^{1}$ Angel J. Moreno, ${ }^{2,3}$ and Christos N. Likos ${ }^{1}$

1 - Faculty of Physics, University of Vienna, Boltzmanngasse 5, A-1090 Vienna, Austria

2 - Centro de Física de Materiales, CSIC-UPV/EHU, Apartado 1072, E-20080 San Sebastián, Spain

3 - Donostia International physics Center, Paseo Manuel de Lardizabal 4, E-20018 San Sebastian, Spain

\section{ABSTRACT}

We present a review of recent computational investigations on the properties of ring polymers in solution. In particular, we focus on effective interactions obtained by means of coarse-graining techniques. We discuss the relative importance of the self-avoidance and the topological contributions in the qualitative features of the effective potential. We extend our previous results on identical rings and determine the effective potential between dissimilar ring polymers of distinct topology and size. The obtained results evidence the dramatic effects of the specific topology on the effective interactions, and hence in the structural correlations, of polymeric systems. 
Multiscale simulations is one of the most active and important fields in many areas of computational science $[1,2]$. They are an indispensable tool to obtain relevant information on a variety of physical systems at different length- and time scales. They find wide applications in, e.g., biological systems [3, 4], in the study of structural correlations [5] or as a means to improve simulation performance in combination with renormalization group theory [6]. Multiscale simulations of complex polymer systems involve the application of coarse-graining (CG) techniques. CG amounts to the systematic elimination of the most local and faster degrees of freedom in the system, allowing the reduction of computational expense and the bridging of different length and time scales. This is particularly useful in polymers, which exhibit a rich and complex structure as well as relaxation processes extending over a extremely broad dynamic window. In the field of biophysics, DNA is an archetypical example of this complexity [7]. Basic biological process, such as DNA replication, involve a vast spectrum of length and time scales, arising from the long double helix structure. Another relevant aspect of the macromolecular complexity is topology. This problem has generated increasing interest on the biophysical community over the last years, since long biopolymers as DNA are easily found in the knotted state [8 - 13]. Knotting is also possible for smaller systems such as proteins [14]. Given the importance of such systems and the role played in them by topology, it is pertinent to pay attention in coarse-grained techniques in which topology as an ingredient from the very outset.

The mathematical object that takes into account the interactions between coarse-grained particles is known as effective potential, $V_{\text {eff }}(R)$ [15]. Here, $R$ is the distance between two suitably chosen degrees of freedom, each one effectively describing the whole molecule. A typical and useful choice is to use the centers of mass of the two considered macromolecules. The knowledge of $V_{\text {eff }}(R)$ is a key goal of CG techniques. The effective potential allows to extract the relevant information in order to simulate polymer solutions at least up to the overlap concentration, removing the irrelevant fast degrees of freedom, i.e., the fluctuations of the individual monomers. An illustrative example of the usefulness of CG is the fact that $V_{\text {eff }}(R)$ is closely related with the second virial coefficient, $B_{2}$, of the solution. The temperature for which $B_{2}=0$ coincides with the so-called $\Theta$-point, i.e., the temperature at which the dissolved polymers behave as ideal (Gaussian conformations). A considerable amount of work has been dedicated to the calculation of $V_{\text {eff }}(R)$ combining different solvent quality and topological conditions, as for e.g. linear polymers $[16,17]$ or unknotted ring polymers $[18,19]$. The relevant determination of $V_{\text {eff }}(R)$ allows the prediction, via the condition $B_{2}=0$, of a decrease of the $\Theta$-temperature for ring polymers with respect to their linear counterparts. This has been confirmed experimentally $[20,21]$.

One of the most striking effects of topology on the effective interactions is given by the so-called topological potential between two unknotted ring polymers, $V_{\text {topo }}(R)$, which is a part of the total contribution to the effective potential $V_{\text {eff }}(R)$. This topological interaction exists even for the ideal case of infinitely thin and non-interacting ring polymers, and it arises from the constraint that, as the two rings approach each other, any concatenation or link formation between them is forbidden. This topological constraint 
gives rise to a reduction of the number of available states, and thus of the total entropy. The resulting topological interaction was first considered in the pioneering work of Frank-Kamenetskii et al. [22], and has been investigated in detail in the recent works of Hirayama et al. [23] and of Bohn and Heermann [24]. There, the interdependence between topological interactions and those stemming from self-avoidance was discussed. Thus, it was shown that $V_{\text {topo }}(R)$ changes with the strength of the excluded-volume parameter and it also depends on the degree of polymerization $N$ of the molecules.

The mentioned studies regard the case of simple circular („unknotted“) rings. To the best of our knowledge, the influence of the knotedness of the rings on the effective potential has only been considered in a recent work by Narros et. al. [25]. In that work the authors derived by Monte Carlo (MC) techniques effective potentials between unknotted rings as well as between knotted rings with different topology. The study was restricted to the case of identical rings (i.e., both rings having the same topology and $N$ ). For moderate values of $N$, it was found that the strength of the effective potential grows with increasing complexity of the knot. In this contribution we extend the investigation of Ref. [25] and we obtain effective potentials between pairs of dissimilar rings (distinct topologies and number of monomers). The results presented below provide new insights into the role of molecular size and toplogical contraints in the properties of ring polymers [18,19,23 - 25].

In our coarse-grained model for ring polymers the relevant magnitudes are the distance between the centers of mass (CM) of the rings, $R$, and their average size characterized by the radius of gyration, $R_{g}$. We have calculated the effective interaction between two ring polymers, whose CMs are at a mutual distance $R$. The topologies of the two rings are denoted by $\tau_{i}$ and their degree of polymerization by $N_{i}$, with $i=A, B$. Thus, we denote the effective potential between $A$ and $B$ as $V_{\text {eff }}\left(R ; \tau_{A}, N_{A}, \tau_{B}, N_{B}\right)$. We consider the simplest ring topologies of torus knots, i.e., the unknot (or trivial knot) $0_{1}$, the trefoil knot, $3_{1}$, and the $5_{1}$-knot. These are very common in nature, because they have high probability to be obtained from a long polymer [26]. Indeed, these topologies are very often found in circular DNA [10, 27], both in dilute and semi-dilute conditions, as well as in proteins [14]. For completeness, we also introduce the notation $\tau=L$ to refer to common linear polymers. We use a model of tethered hard-spheres for the ring polymers, as described in Ref. [25]. The maximum allowed values of the bond length and MC step are selected in order to prevent accidental concatenation by bond-crossing [25]. Following the procedure described in Ref. [25], we have used the umbrella-sampling MC technique to generate configurations of the two rings at the whole relevant range of CM-distances. This procedure allows us to obtain the radial distribution function $g(R)$ with high accuracy. The effective potential is then obtained as [25] $V_{e f f}(R)=-k_{B} T \ln g(R)$, with $T$ the temperature and $k_{B}$ Boltzmann's constant.

The average radius of gyration $R_{g}$ of an isolated ring polymer in equilibrium depends on both the topology $\tau$ and the monomer number $N$ and it follows the law [28]: 


$$
R_{g}^{2}(\tau, N) \propto\left\{\begin{array}{cc}
N^{2 v_{0}} & N \leq N^{*}(\tau) \\
N^{2 v} & N \leq N^{*}(\tau),
\end{array}\right.
$$

where $v_{0}=1 / 2$ and $v \cong 3 / 5$ are the critical exponents of the random walk and the self-avoiding random walk, respectively, and $N^{*}(\tau)$ is a topology- and model-dependent crossover value of $N$. Typically, $N^{*}\left(0_{1}\right) \cong 300$, for unknotted rings whereas this value grows for more complicated complex knotted topologies. Due to computational limitations, we have used relatively short molecules, i.e., $N \in[15,200]$. When two rings interact with each other, the gyration radii are in general a function of their separation $R$ : $R_{g, i}=R_{g, i}\left(R ; \tau_{A}, N_{A}, \tau_{B}, N_{B}\right), i=A, B$. Obviously, for infinite separation the latter is identical to the undisturbed gyration radius of an isolated ring (Eq. (1)). We thus introduce the notation:

$$
R_{g, i}\left(R \rightarrow \infty ; \tau_{A}, N_{A}, \tau_{B}, N_{B}\right) \equiv R_{g}^{0}\left(\tau_{i}, N_{i}\right) .
$$

Accordingly, we also define a "swelling factor", $\alpha_{i}$, as the relative change of the size of the ring with respect to its undisturbed size $(R \rightarrow \infty)$, due to its interation with the other ring when their centers of mass coincide $(R=0)$ :

$$
\alpha_{i}\left(\tau_{A}, N_{A}, \tau_{B}, N_{B}\right) \equiv \frac{R_{g, i}\left(R=0 ; \tau_{A}, N_{A}, \tau_{B}, N_{B}\right)}{R_{g}^{0}\left(\tau_{i}, N_{i}\right)} .
$$

In the following, the distance between the CM's of the two rings will be measured in units of the molecule-averaged infinite-separation radius of gyration:

$$
\left\langle R_{g}^{0}\right\rangle \equiv \frac{R_{g}^{0}\left(\tau_{A}, N_{A}\right)+R_{g}^{0}\left(\tau_{B}, N_{B}\right)}{2} .
$$

The effective potential $V_{\mathrm{eff}}(R ; L, N, L, N)$ between two linear polymers of $N$ monomers each has a characteristic Gaussian shape, a result arising both from on-lattice $[16,24,29]$ and off-lattice simulations [25] at good solvent conditions. This behavior can be rationalized by the fact that the monomer distribution around the center of mass is approximately Gaussian for a linear chain. For sufficiently long chains, the effective potential becomes a universal function of $R /\left\langle R_{g}^{0}\right\rangle$, and it has an $N$-independent amplitude $V_{\text {eff }}(R=0 ; L, N, L, N) \cong 2 k_{\mathrm{B}} T$ at full overlap [16, 24, 25], in agreement with theoretical predictions [30,31]. The bounded value at full overlap is a general feature of polymers, stemming from their property of being inter-penetrable, which allows for coincidence of the centers-of-mass without violation of monomer excluded volume constraints. The bounded character of the effective potential also holds for -ring polymers $[24,25]$ though in this case its amplitude is higher than for linear polymers, namely $V_{\mathrm{eff}}\left(R=0 ; 0_{1}, N, 0_{1}, N\right) \cong 6 k_{\mathrm{B}} T$. In other words, the effective repulsion between polymers with the same $N$ is much stronger for rings than for linear chains. However, only a small fraction of this repulsive interaction can be attributed to the topological potential [22 - 24].

The specific molecular topology does not only alter the effective potential quantitatively. It also leads to qualitative differences. In particular, the effective potential $V_{\text {eff }}(R)$ for $0_{1}$-ring polymers does not show the Gaussian form found for their linear 
counterparts [23 - 25]. Its shape rather features a plateau at separations $R \leq 0.5\left\langle R_{g}^{0}\right\rangle$ (see Fig. 1), and even a minimum at zero separation [25] for small rings (reflecting an effective short-range attraction). This feature stems from the typical configurations of two unknotted rings at small mutual separation $(R \rightarrow 0)$, in which one of the rings adopts and open conformation that facilitates interpenetration [25]. Similar features have been observed in the effective potential for amphiphilic dendrimers [32], giving rise to clustered structures under certain conditions [33].

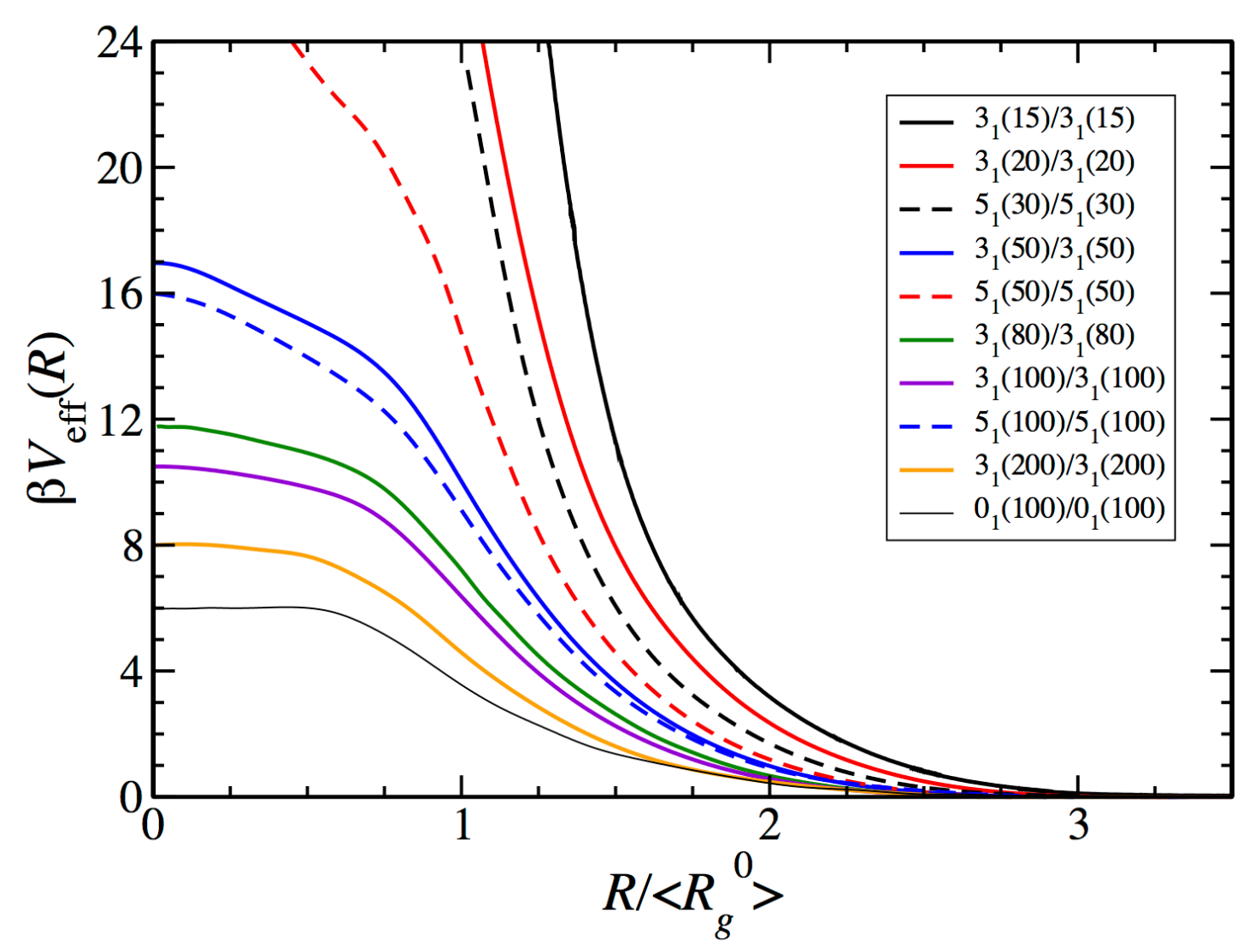

Figure 1: The effective potential $V_{\text {eff }}(R)$ for identical ring polymers of $3_{1}$ (solid lines) and $5_{1}$ (dashed lines) topologies, and for different degrees of polymerization $N$ (see parentheses in the legend). The effective potential for $0_{1}$-rings is included for comparison (lower thin solid line).

Let us now discuss effective potentials for knotted rings, namely with $3_{1}$ - and $5_{1}$ -topologies. Fig. 1 shows results for $V_{\text {eff }}\left(R ; 3_{1}, N, 3_{1}, N\right)$ and $V_{\text {eff }}\left(R ; 5_{1}, N, 5_{1}, N\right)$. As can be seen, the scaling regime has not been reached neither for $3_{1}$ nor for $5_{1}$-rings for $N$ as large as 100, within the investigated range of polymerization degree $N \leq 200$, in contrast 
to the case of $0_{1}$-topology, for which the effective potential already approaches a universal form for $N \approx 80$ [25]. Nevertheless, there is an apparent trend, especially for the $3_{1}$-case, towards convergence to the universal form obtained for the $0_{1}$-topology. For the smallest values of $N$, a strongly repulsive potential is found for both $3_{1}$ - and $5_{1}$-topologies. This is an expected result since knotting yields smaller and thus denser molecules, giving rise to stronger self-avoidance effects. Such effects diminish for larger rings, in which the knot size becomes negligible in comparison to the overall size of the ring. Since $V_{\text {eff }}\left(R ; 5_{1}, N, 5_{1}, N\right)>V_{\text {eff }}\left(R ; 3_{1}, N, 3_{1}, N\right)$ we expect that the convergence to the scaling limit will be reached at smaller $N$ for $3_{1}$-rings than for $5_{1}$-rings. This guess is consistent with the observation that $N^{*}\left(5_{1}\right)>N^{*}\left(3_{1}\right)$ for the crossover (Eq.(1)) in the scaling behavior of $R_{g}^{0}$.

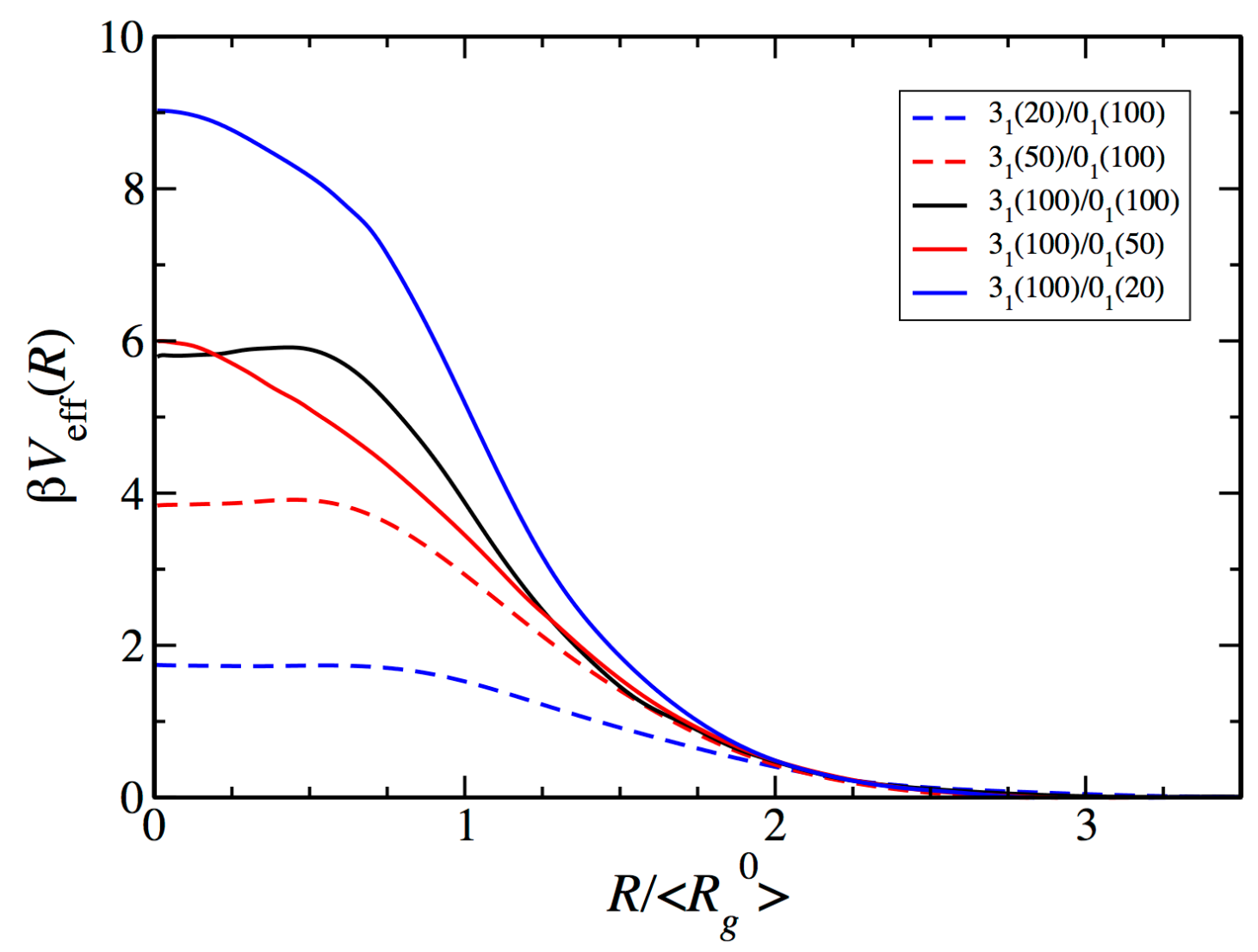

Figure 2: The effective potentials $V_{\text {eff }}\left(R ; 3_{1}, 100,0_{1}, N\right)$ (solid lines) and $V_{\text {eff }}\left(R ; 0_{1}, 100,3_{1}, N\right)$ (dashed lines). The molecular sizes for the $B$ molecule are specified in the legend.

New results for dissimilar rings, $V_{\text {eff }}\left(R ; 3_{1}, 100,0_{1}, N\right)$ and $V_{\text {eff }}\left(R ; 0_{1}, 100,3_{1}, N\right)$, are shown in Fig. 2. For the cases of $V_{\text {eff }}\left(R ; 3_{1}, 100,0_{1}, 20\right)$ and $V_{\text {eff }}\left(R ; 3_{1}, 100,0_{1}, 50\right)$, there is no 
plateau or a minimum at $R=0$, i.e., the interaction becomes more repulsive the smaller the separation between centers-of-mass. A tentative explanation for this feature is that the large knotted ring has to open up in order to be penetrated by the small unknotted ring. However this involves localization of the knot, which is entropically very unfavorable. On the contrary, a plateau, and even a minimum at zero separation, are clearly visible for the case $V_{\text {eff }}\left(R ; 3_{1}, 100,0_{1}, 100\right)$. Inspections of the molecular conformations (see Fig. 3 for a representative case), reveal that in this case the large unknotted ring adopts an open conformation and is penetrated by the small knotted ring, in analogy with the observation for two identical unknotted rings [25]. This feature is also reflected in the behavior of the swelling factors $\alpha_{i}, i=A, B$, defined in Eq. (3). Thus, we find $\alpha_{B}\left(3_{1}, 100,0_{1}, 100\right) \cong 1.57$, i.e., a swelling of the unknotted by almost $60 \%$, to accomodate the $3_{1}$-molecule, whilst the latter retains its size, $\alpha_{A}\left(3_{1}, 100,0_{1}, 100\right) \cong 1$. Interestingly, the swelling of the $0_{1}$-ring is present in the whole range of $N$ for which the effective potential exhibits a plateau or a short-range attraction. Concomitantly, the knotted ring maintains its size in the former range of $N$. Indeed, interpenetration facilitated by swelling of the knotted ring is entropically unfavourable, since it would involve localization of the knot (see also above). Since the $0_{1}$-ring is already big enough, no strong swelling of the latter is necessary at full interpenetration. For instance, we find $\alpha_{A}\left(0_{1}, 100,3_{1}, 20\right) \cong 1.17$ and $\alpha_{B}\left(0_{1}, 100,3_{1}, 20\right) \cong 1$ for the unknotted and knotted ring, respectively. Finally, due to the high penalty for knotted rings to swell, penetration of large unknotted rings by small knotted rings is more favourable than penetration of large knotted rings by small unknotted rings. Hence, the effective potential $V_{\text {eff }}\left(R ; 0_{1}, 100,3_{1}, N\right)$ is weaker than its counterpart $V_{\text {eff }}\left(R ; 3_{1}, 100,0_{1}, N\right)$.

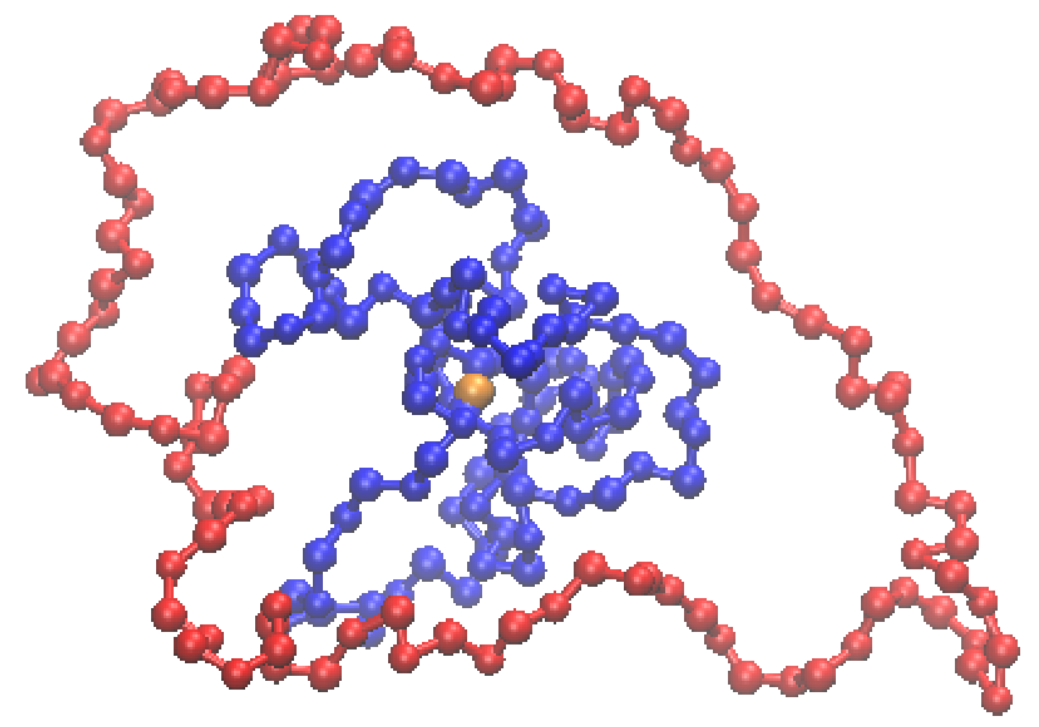

Figure 3: A typical simulation snapshot showing a $3_{1}$ - (blue) and a $0_{1}$ - (red) ring polymer of $N=100$ monomers each, with their centers of mass coinciding (golden bead in the middle).

It is pertinent to ask what is the contribution of the topological interaction to the total effective potential. For $0_{1}$-rings, it has been shown that the topological contribution 
amounts to less that $10 \%$ of the full interaction potential $[23,24]$. Despite its rather small magnitude, the topological contribution has the particular feature of showing an attractive part at small distances, in contrast to the monotonic repulsive contribution that arises from self-avoidance. The interplay of both contributions brings about a plateau, and even a minimum at $R=0$ for the full $V_{\text {eff }}\left(R ; 0_{1}, N, 0_{1}, N\right)$ [25]. As regards the cases examined here, we find that for small identical knotted rings the effect of the topological potential is even smaller, since the steric crowding caused by the knots dominates the effective interaction. However, as $N$ grows, $V_{\text {eff }}(R ; \tau, N, \tau, N)$ seems to converge to a form independent of the topology, i.e, the relative importance of the topological and self-avoidance terms will approach the one discussed for the $0_{1}$-case. For dissimilar rings, the topological contribution to $V_{\text {eff }}(R)$ seems to be even less important: when the large ring is the knotted one there is no plateau in $V_{\text {eff }}(R)$, and in the opposite case the weak swelling of the large unknotted ring is sufficient to explain the existence of the plateau on the basis of excluded-volume interactions alone [34].

We have put forward a concise review of previously derived results for the effective interaction between identical ring polymers in good solvent. Moreover we have extended this work to derive effective interactions between dissimilar rings, of different sizes and topologies. We have assigned salient characteristics of the derived potentials to the relative swelling of the rings at small separation. A short-range attraction in the effective potential is found when the large ring is the unknotted one, whereas a monotonic repulsion is observed in the opposite case. The obtained results suggest that the topological contribution to the effective potential is less important for knotted rings than for unknotted ones. Consequently, we expect that the spatial correlations between, e.g., knotted proteins or DNA molecules in concentrated environments are dominated by the knotting of the individual polymers and not by an influence of the knots on the constraint of no concatenation between any two molecules.

We acknowledge helpful discussions with Ronald Blaak. This work has been supported by the European Community's Seventh Framework Programme (FP7/2007-2013) under the IEF-RINGEFF, Grant Agreement 236664 and by the Austrian Science Fund (FWF), Grant 23400-N16.

\section{References}

1 S. Baeurle (2009): Multiscale modeling of polymer materials using field-theoretic methodologies: a survey about recent developments. J. Math. Chem. 46:363-426.

2 J. J. de Pablo (2011): Coarse-grained simulations of macromolecules: From DNA 
to nanocomposites. Annu. Rev. Phys. Chem., 62(1):555-574.

3 S. Izvekov and G. A. Voth (2005): A multiscale coarse-graining method for biomolecular systems. J. Phys. Chem. B, 109(7):2469-2473.

4 S. Izvekov and G. A. Voth (2005): Multiscale coarse graining of liquid-state systems. J. Chem. Phys., 123(13):134105.

5 W. G. Noid, J.-W. Chu, G. S. Ayton, and G. A. Voth. (2007): Multiscale coarse-graining and structural correlations: Connections to liquid-state theory. J. Phys. Chem. B, 111(16):4116-4127, 2007.

6 N. Goldenfeld, B. Athreya, and J. Dantzig (2006): Renormalization group approach to multiscale modelling in materials science. J. Stat. Phys., 125:1015-1023.

7 Y.-W. Kwon, C. H. Lee, D.-H. Choi, and Jung-Il Jin (2009): Materials science of DNA: J. Mater. Chem., 19(10):1353-1380.

8 J. P. J. Michels and F. W. Wiegel (1982): Probability of knots in a polymer ring. Phys. Lett. A, 90(7):381-384.

9 M. K. Shimamura and T. Deguchi (2002): Knot complexity and the probability of random knotting. Phys. Rev. E, 66(4):040801.

10 V. V. Rybenkov, N. R. Cozzarelli, and A. V. Vologodskii (1993): Probability of DNA knotting and the effective diameter of the dna double helix. Proc. Nat. Acad. Sci. U.S.A., 90(11):5307-5311.

11 K. C. Neuman (2010): Single-molecule measurements of DNA topology and topoisomerases. J. Bio. Chem., 285(25):18967-18971.

12 J. Arsuaga, M. Vázquez, S. Trigueros, D. W. Sumners, and J. Roca (2002): Knotting probability of DNA molecules confined in restricted volumes: DNA knotting in phage capsids. Proc. Nat. Acat. Sci. U.S.A., 99(8):5373-5377.

13 X. R. Bao, H. J. Lee, and S. R. Quake (2003): Behavior of complex knots in single DNA molecules. Phys. Rev. Lett., 91(26):265506.

14 J. I. Sukowska, E. J. Rawdon, K. C. Millett, J. N. Onuchic, and A. Stasiak (2012): Conservation of complex knotting and slipknotting patterns in proteins. Proc. Nat. Acad. Sci., U.S.A., 109(26):E1715-E1723.

15 C. N. Likos (2001): Effective interactions in soft condensed matter physics. Phys. Reports, 348(1):267-439.

16 V. Krakoviack, J.-P. Hansen, and A. A. Louis (2003): Influence of solvent quality on effective pair potentials between polymers in solution. Phys. Rev. E, 67(4):041801. 
17 I. M. Withers, A. V. Dobrynin, M. L. Berkowitz, and M. Rubinstein (2003): Monte carlo simulation of homopolymer chains. i. second virial coefficient. J. Chem. Phys., 118(10):4721-4732.

18 F. Tanaka (1987): Osmotic pressure of ring-polymer solutions, J. Chem. Phys., 87(7):4201-4206.

19 K. Iwata (1989): Theta temperature of ring polymers: another evidence of topological interaction. Macromolecules, 22(9):3702-3706.

20 J. Roovers (1988): Viscoelastic properties of polybutadiene rings. Macromolecules, 21(5):1517-1521.

21 G. B. McKenna, G. Hadziioannou, P. Lutz, G. Hild, C. Strazielle, C. Straupe, P. Rempp, and A. J. Kovacs (1987): Dilute solution characterization of cyclic polystyrene molecules and their zero-shear viscosity in the melt. Macromolecules, 20(3):498-512.

22 M. D. Frank-Kamenetskii, A. V. Lukashin, and A. V. Vologodskii (1975): Statistical mechanics and topology of polymer chains. Nature, 258(5534):398-402.

23 N. Hirayama, K. Tsurusaki, and T. Deguchi (2009): Linking probabilities of off-lattice self-avoiding polygons and the effects of excluded volume. J. Phys. A:Math. Gen., 42(10):105001.

24 M. Bohn and D. W. Heermann (2010): Topological interactions between ring polymers: Implications for chromatin loops. J. Chem. Phys., 132(4):044904.

25 A. Narros, A. J. Moreno, and C. N. Likos (2010): Influence of topology on effective potentials: coarse-graining ring polymer. Soft Matter, 6(11):2435-2441.

26 C. Micheletti, D. Marenduzzo, E. Orlandini, and D. W. Sumners (2006): Knotting of random ring polymers in confined spaces. J. Chem. Phys., 124(6):064903-10.

27 D. Buck (2009): DNA topology. Proc. Symp. Appl. Math., 66, 2009.

28 C. Micheletti, D. Marenduzzo, and E. Orlandini (2011): Polymers with spatial or topological constraints: Theoretical and computational results. Phys. Reports, 504:1-73.

29 C. Pierleoni, C. Addison, J.-P. Hansen, and V. Krakoviack (2006): Multiscale coarse graining of diblock copolymer self-assembly: From monomers to ordered micelles. Phys. Rev. Lett., 96(12):128302.

30 M. Daoud, J. P. Cotton, B. Farnoux, G. Jannink, G. Sarma, H. Benoit, C. Duplessix, C. Picot, and P. G. de Gennes (1975): Solutions of flexible polymers. Neutron experiments and interpretation. Macromolecules, 8(6):804-818. 
31 A. Y. Grosberg, P. G. Khalatur, and A. R. Khokhlov (1982). Polymeric coils with excluded volume in dilute solution: The invalidity of the model of impenetrable spheres and the influence of excluded volume on the rates of diffusion-controlled intermacromolecular reactions. Makromol. Chem. Rapid Commun., 3(10):709-713.

32 B. M. Mladek, G. Kahl, and C. N. Likos (2008): Computer assembly of cluster-forming amphiphilic dendrimers. Phys. Rev. Lett., 100(2):028301.

33 D. A. Lenz, B. M. Mladek, C. N. Likos, G. Kahl, and R. Blaak (2011): Monomer-resolved simulations of cluster-forming dendrimers. J. Phys. Chem. B, 115:7218-7226.

34 A. Narros, A. J. Moreno, and C. N. Likos. An overlapping-spheres model for the effective interaction between ring polymers (in preparation). 